\title{
Visible-Light-Mediated Oxidative Debenzylation Enables the Use of Benzyl Ethers as Temporary Protecting Groups
}

\author{
Cristian Cavedon, Eric T. Sletten, Amiera Madani, Olaf Niemeyer, Peter H. Seeberger,* \\ and Bartholomäus Pieber*
}

Cite This: Org. Lett. 2021, 23, 514-518

Read Online

\section{ACCESS | Lill Metrics \& More | 回 Article Recommendations ｜（） Supporting Information}

ABSTRACT: The cleavage of benzyl ethers by catalytic hydrogenolysis or Birch reduction suffers from poor functional group compatibility and limits their use as a protecting group. The visible-light-mediated debenzylation disclosed here renders benzyl ethers temporary protective groups, enabling new orthogonal protection strategies. Using 2,3-dichloro-5,6-dicyano-1,4benzoquinone (DDQ) as a stoichiometric or catalytic photooxidant, benzyl ethers can be cleaved in the presence of azides, alkenes, and alkynes. The reaction time can be reduced from hours to minutes in continuous flow.

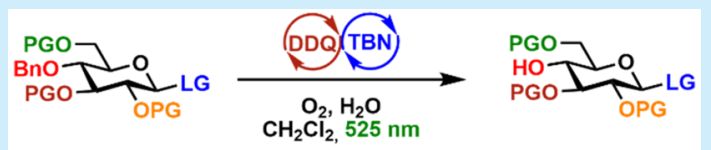

- Selective debenzylation

- Mild conditions

- High functional group tolerance

$\mathrm{T}$ he synthesis of complex molecules such as biopolymers relies on protective groups to ensure chemo-, regio-, and stereoselectivity. ${ }^{1}$ Protecting groups are of central importance to carbohydrate construction, where a host of hydroxyl groups have to be masked. Installation and selective removal are the basis for orthogonal protecting group strategies that are key to the synthesis of well-defined oligosaccharides. ${ }^{2-4}$ Benzyl ethers are stable over a wide range of conditions, making them an ideal protecting group that is removed only at the very end of the synthesis. ${ }^{1}$ For this very reason, however, benzyl ether cleavage requires harsh reduction/oxidation processes, such as catalytic hydrogenolysis, Birch reduction, or oxidation with ozone or $\mathrm{BCl}_{3}$, which are incompatible with many functional groups $^{1,5}$ and are hazardous. ${ }^{6,7}$ Methods for the mild and selective cleavage of benzyl ethers would render them attractive temporary protective groups that would conceptually change the strategic approach toward the synthesis of complex glycans.

Compared with benzyl ethers, p-methoxybenzyl (PMB) ethers can be selectively cleaved using mild stoichiometric oxidants. ${ }^{1,8-10}$ Photoredox catalysis was used to selectively cleave PMB ethers (Scheme 1a). ${ }^{11-13}$ Benzyl ethers $\left(E_{\mathrm{Bn}-\mathrm{O}-\mathrm{Me}}\right.$ $=2.20 \mathrm{~V}$ vs saturated calomel electrode $\left.(\mathrm{SCE})^{14}\right)$ have a significantly higher oxidation potential compared with $\mathrm{PMB}$ ethers $\left(E_{\mathrm{PMB}-\mathrm{O}-\mathrm{Me}}=1.60 \mathrm{~V}\right.$ vs $\left.\mathrm{SCE}^{14}\right)$ and are stable during the photocatalytic PMB cleavage. ${ }^{11-13}$

A photocatalyst (PC) with a sufficiently strong oxidizing excited state could facilitate the oxidative cleavage of benzyl ethers with high functional group tolerance (Scheme 1b). To move this concept to practice, a suitable $\mathrm{PC}, \mathrm{H}$-atom acceptor, and terminal oxidant had to be identified using the debenzylation of $\mathrm{C}(3)$-O-benzyl-tetraacetylglucoside (1a) as a model reaction. Initial efforts with common PCs were not successful. A combination of $4 \mathrm{~mol} \%$ 9-mesityl-10methylacridinium as the $\mathrm{PC}$ and 5.0 equiv of $\mathrm{CBr}_{4}$, for
Scheme 1. Visible-Light-Mediated Oxidative Deprotection Strategies of PMB and Benzyl Ethers

a) photocatalytic deprotection of PMB ethers (ref. 11)

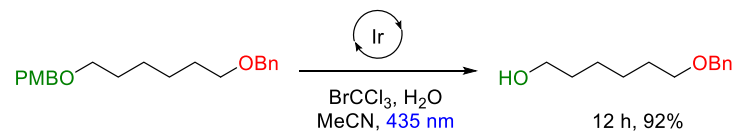

b) this work: selective photocatalytic deprotection of benzyl ethers

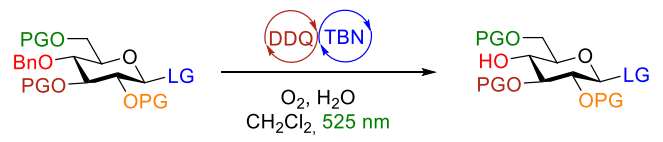

example, was capable of hydrolyzing the benzyl ether, but concomitant product degradation resulted in low yields (Table S2).

Full conversion of the starting material and excellent selectivity toward the desired product $(\mathbf{1 b})$ were achieved using stoichiometric amounts of 2,3-dichloro-5,6-dicyano-1,4benzoquinone (DDQ) $\left(E^{3} \mathrm{DDQ}^{*} / \mathrm{DDQ}^{-\bullet}=3.18 \mathrm{~V}\right.$ vs $\left.\mathrm{SCE}^{15}\right)$ and green-light irradiation $(525 \mathrm{~nm})$ in wet dichloromethane (Table 1 , entry 1$)$. In contrast with photochemical PMB deprotection, ${ }^{11-13}$ an additional $\mathrm{H}$-atom acceptor is not required, as the single electron transfer oxidation and the hydrogen abstraction are executed by DDQ upon irradiation. ${ }^{16}$

Received: December 4, 2020

Published: January 5, 2021 
Table 1. Optimized Conditions and Control Experiments for the Visible-Light-Mediated Oxidative Debenzylation Using Catalytic and Stoichiometric Amounts of $\mathrm{DDQ}^{a}$

\begin{tabular}{|c|c|c|c|c|c|c|}
\hline \multirow[t]{2}{*}{$\underset{\substack{\mathrm{OAc} \\
\mathbf{1 a}}}{\mathrm{maAc}}$} & \multicolumn{2}{|c|}{$\frac{\text { Protocol A or B }}{\underset{\mathrm{CH}_{2} \mathrm{Cl}_{2} / \mathrm{H}_{2} \mathrm{O}(99: 1)}{525 \mathrm{~nm}}}$} & \multicolumn{2}{|c|}{${ }_{\mathbf{O A C}}$} & $\begin{array}{c}\mathrm{AcO}- \\
\mathrm{AcO} \\
\mathrm{AzO}\end{array}$ & $\frac{\mathrm{DO}}{\mathrm{OAC}}$ \\
\hline & entry & variation & time & $\begin{array}{c}\text { 1a } \\
{[\%]^{b}}\end{array}$ & $\begin{array}{c}\mathbf{1 b} \\
{[\%]^{\mathrm{b}}}\end{array}$ & $\begin{array}{c}\mathbf{1 c} \\
{[\%]^{\mathrm{b}}}\end{array}$ \\
\hline & 1 & none & $30 \mathrm{~min}$ & n.d. ${ }^{c}$ & 96 & $<2$ \\
\hline $\mathrm{O}_{2}$-free & 2 & $440 \mathrm{~nm}$ & $10 \mathrm{~min}$ & n.d. & 78 & 16 \\
\hline 1.5 & 3 & no light & $24 \mathrm{~h}$ & 99 & n.d. & n.d. \\
\hline & 4 & none & $2 \mathrm{~h}$ & n.d. & 97 & $<2$ \\
\hline & 5 & $440 \mathrm{~nm}$ & $30 \mathrm{~min}$ & n.d. & 87 & 11 \\
\hline TBN air & 6 & no light & $2 \mathrm{~h}$ & 98 & $<2$ & n.d. \\
\hline $25 \mathrm{~mol} \% 25 \mathrm{~mol} \%$ & 7 & no DDQ & $2 \mathrm{~h}$ & 100 & n.d. & n.d. \\
\hline & 8 & no TBN & $2 \mathrm{~h}$ & 77 & 23 & n.d. \\
\hline & 9 & degassed & $2 \mathrm{~h}$ & 57 & 40 & $<2$ \\
\hline
\end{tabular}

${ }^{a}$ Reaction conditions: 1a $(50 \mu \mathrm{mol})$, DDQ (Protocol A: $75 \mu \mathrm{mol}$, Protocol B: $12.5 \mu \mathrm{mol}$ ), TBN (Protocol B: $12.5 \mu \mathrm{mol}$ ), $\mathrm{CH}_{2} \mathrm{Cl}_{2}(5$ $\mathrm{mL}), \mathrm{H}_{2} \mathrm{O}(50 \mu \mathrm{L}), 525 \mathrm{~nm}$ irradiation at $\mathrm{rt} .{ }^{b}$ Determined by ${ }^{1} \mathrm{H}$ NMR using maleic acid as the internal standard. ${ }^{c}$ Not detected.

The irradiation source is crucial for achieving high selectivity. Shorter wavelengths $(440 \mathrm{~nm})$ result in the formation of significant amounts of the corresponding benzoyl ester 1c (entry 2). The cleavage of benzyl ethers using simple substrates was previously reported using stoichiometric amounts of DDQ under UV irradiation but suffered from low functional group compatibility. ${ }^{17}$

To avoid the tedious separation of the stoichiometric byproduct 2,3-dichloro-5,6-dicyano-1,4-hydroquinone $\left(\mathrm{DDQH}_{2}\right)$, we ultimately developed a catalytic protocol using DDQ (25 mol \%), tert-butyl nitrite (TBN, $25 \mathrm{~mol} \%$ ) as the cocatalyst, and air as the terminal oxidant (Table 1, entry 4). ${ }^{18-25}$ The nitrite thermally or photochemically releases $\mathrm{NO}$ that is oxidized by $\mathrm{O}_{2}$ to $\mathrm{NO}_{2}$ and reoxidizes $\mathrm{DDQH}_{2}$ to DDQ. ${ }^{16}$ Similar to the protocol with stoichiometric amounts of DDQ, lower selectivities were observed at shorter wavelengths (entry 5). Control studies confirmed that photons and DDQ are necessary for productive catalysis (entries 3, 6, and 7). Monitoring the reaction using an LED-NMR setup supported the notion that the reaction ceases upon light source removal (Figure 1a). ${ }^{26}$ When DDQ is used in catalytic amounts and no TBN is added, the reaction stops after one turnover (Table 1, entry 8). The late addition of TBN can restore $\mathrm{DDQ}$ and the reaction smoothly proceeds until completion (Figure 1b). Under anaerobic conditions, the reaction did not go to completion, confirming that $\mathrm{O}_{2}$ is required (Table 1 , entry 9 ).

Both protocols were evaluated using carbohydrate substrates that carry multiple protecting groups (Scheme 2). The protocol using catalytic amounts of DDQ (protocol B) was slightly modified ( 2 equiv of $\mathrm{TBN}$ ) to avoid long reaction times. Substrates containing acetyl, isopropylidine, and benzoyl protecting groups $(\mathbf{1 a - 4 a})$ were smoothly deprotected in $<4 \mathrm{~h}$ using both protocols and were isolated in excellent yield (84$96 \%)$. Thioethers that could potentially poison palladium catalysts during hydrogenolysis were unproblematic using both

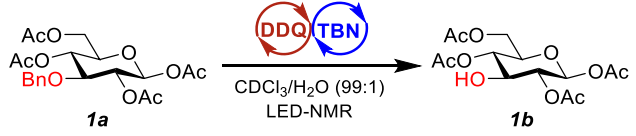

a) light on/off experiment

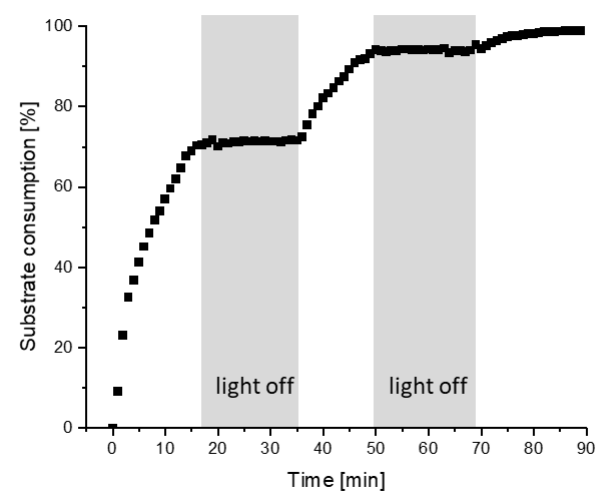

b) delayed TBN addition

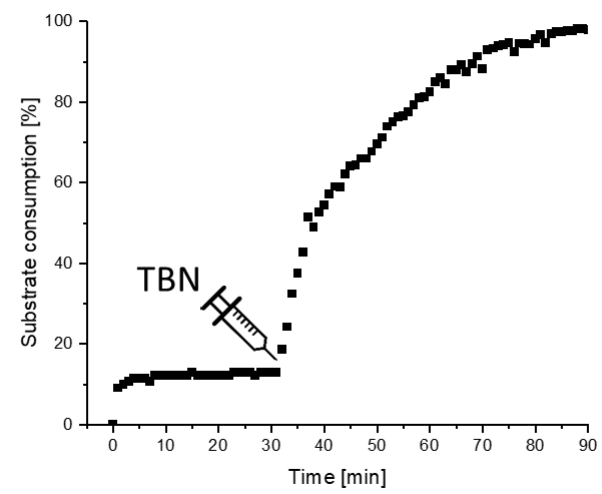

Figure 1. In situ NMR studies using an LED-NMR setup. For experimental details, see the Supporting Information.

photooxidative protocols, and no sulfoxide or sulfone side products were identified $(\mathbf{5 a}-\mathbf{1 1} \mathbf{1})$. Several common protecting groups that are not tolerated in hydrogenolysis or Birch reduction, such as fluorenylmethoxycarbonyl (6a, 7a, 8a), levulinic ester (8a), allyl carbonate (9a), propargyl carbonate (10a), and benzylidene (12a), were well tolerated. Azides (11a), which are essential for biorthogonal labeling, are stable to the photooxidative benzyl ether cleavage. 2-Naphtylmethyl ether (NAP, 12a) is routinely removed using stoichiometric amounts of DDQ in the absence of light. The light-mediated protocol using $25 \mathrm{~mol} \% \mathrm{DDQ}$ (protocol B) provides a valuable alternative to avoid stoichiometric amounts of organic oxidant. The benzyloxycarbonyl ( $\mathrm{Cbz}$ ) group was partially cleaved using stoichiometric amounts of DDQ (protocol A), resulting in a modest isolated yield of the desired product $\mathbf{1 3 b}$. Using the catalytic method (protocol B), longer reaction times resulted in significant cleavage of the $\mathrm{Cbz}$ group. (See the Supporting Information.) Phenylselenyl (14a) and tertbutyldimethylsilyl (TBS, 15a $)^{27}$ groups are not stable under the conditions applied. Whereas the photocatalytic protocol enables the use of benzyl ethers as temporary protective groups, it is not the method of choice to globally deprotect carbohydrates. Full deprotection of perbenzylated glucose 
Scheme 2. Substrate Scope and Limitations for the Visible-Light-Mediated Oxidative Cleavage of Benzyl Ethers ${ }^{a}$

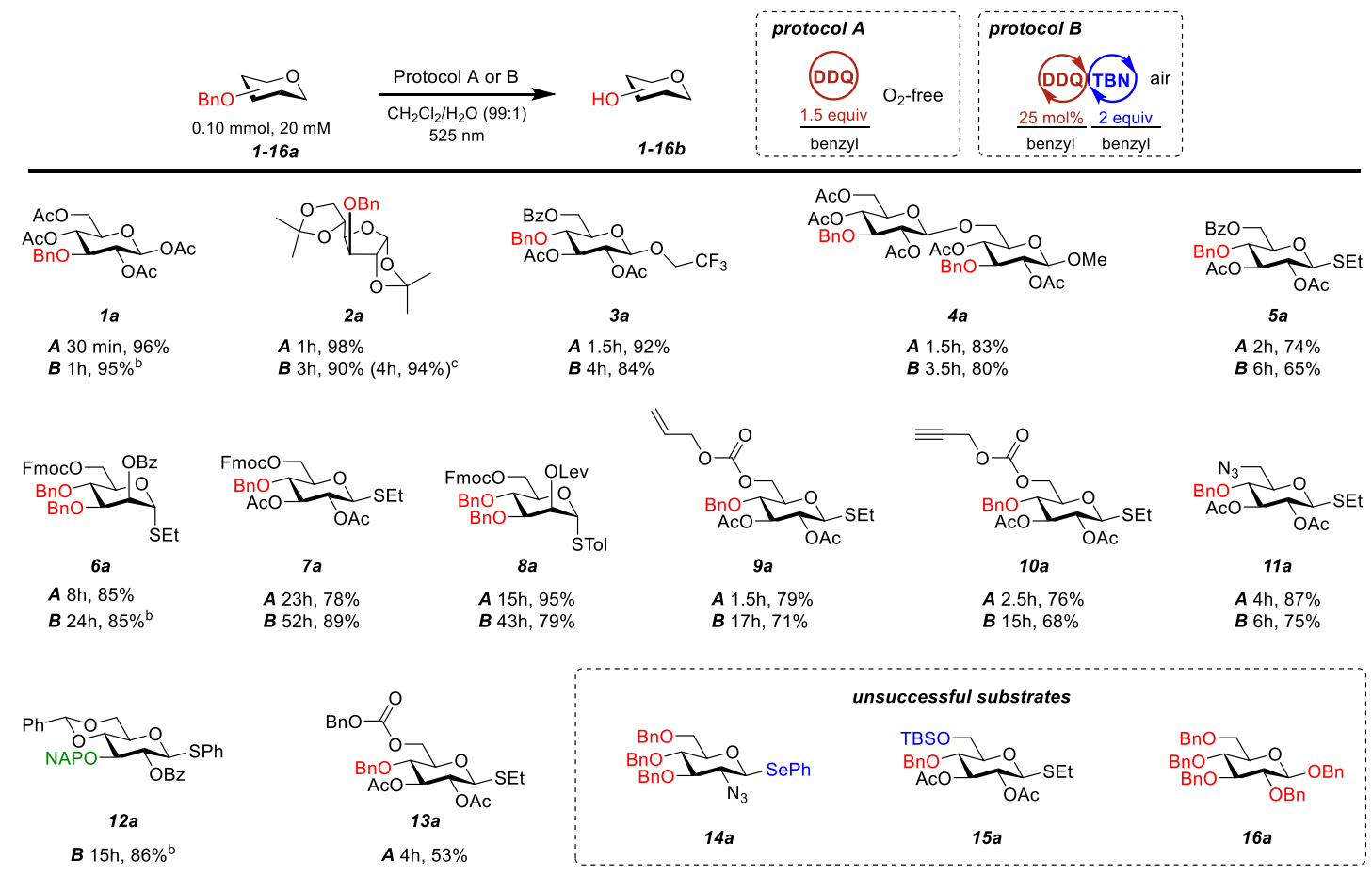

${ }^{a}$ Reaction conditions: benzyl ether $(100 \mu \mathrm{mol})$, DDQ (protocol A: $150 \mu \mathrm{mol} /$ benzyl, protocol B: $25 \mu \mathrm{mol} /$ benzyl), TBN (protocol B, $200 \mu \mathrm{mol}$ ), $\mathrm{CH}_{2} \mathrm{Cl}_{2}(5 \mathrm{~mL}), \mathrm{H}_{2} \mathrm{O}(50 \mu \mathrm{L}), 525 \mathrm{~nm}$ irradiation at rt. ${ }^{b}$ Reaction on a $50 \mu$ mol scale. ${ }^{c}$ Reaction on a $1.5 \mathrm{mmol}$ scale. Isolated yields are reported.

(16a) was not feasible, and a complex mixture of partially deprotected derivatives precipitated during the reaction.

The relatively long reaction times for some substrates are a major limitation, especially using the catalytic protocol. This is a result of the long wavelengths used, as DDQ absorbs only weakly above $450 \mathrm{~nm}$ (Figure S9). When a $440 \mathrm{~nm}$ irradiation source was applied, we observed significantly shorter reaction times but had severe selectivity issues due to overoxidation and product degradation.

Slowing down a chemical reaction to avoid selectivity problems is a common strategy in batch. Continuous-flow chemistry can help to overcome selectivity issues, as it offers precise control over the reaction time and better irradiation. $^{28,29}$ A two-feed setup introduced the homogeneous reaction mixture and air into the reactor unit, which consisted of fluorinated ethylene propylene (FEP) tubing $(0.8 \mathrm{~mm}$ i.d.) and a $440 \mathrm{~nm}$ light source. A short optimization study using C(3)-O-benzyl-glucofuranose $\mathbf{2 a}$ resulted in a significant reduction of the reaction time $(2.5 \mathrm{~min}$ in flow at $440 \mathrm{~nm}$ versus $3 \mathrm{~h}$ in batch at $525 \mathrm{~nm}$ ) while maintaining excellent selectivity (Figure 2a). An experiment using a longer residence time showed that selectivity issues indeed arise from prolonged reaction times at low wavelengths (Figure $2 \mathrm{~b}$ )

The flow approach was subsequently tested for other substrates (Figure 2c). The reaction time for the debenzylation of $10 \mathrm{a}$ was significantly reduced to $3 \mathrm{~min}$, whereas dibenzylated compounds $4 \mathrm{a}$ and $\mathbf{6 a}$ required $10 \mathrm{~min}$.

In conclusion, we developed a mild, photocatalytic debenzylation protocol that is significantly more functionalgroup-tolerant than traditional methods. The proper choice of irradiation source is crucial for reaching high selectivities of benzyl ether cleavage in batch. Green-light irradiation (525 $\mathrm{nm})$ was superior over blue light $(440 \mathrm{~nm})$ in suppressing the formation of side products during batch reactions. A biphasic
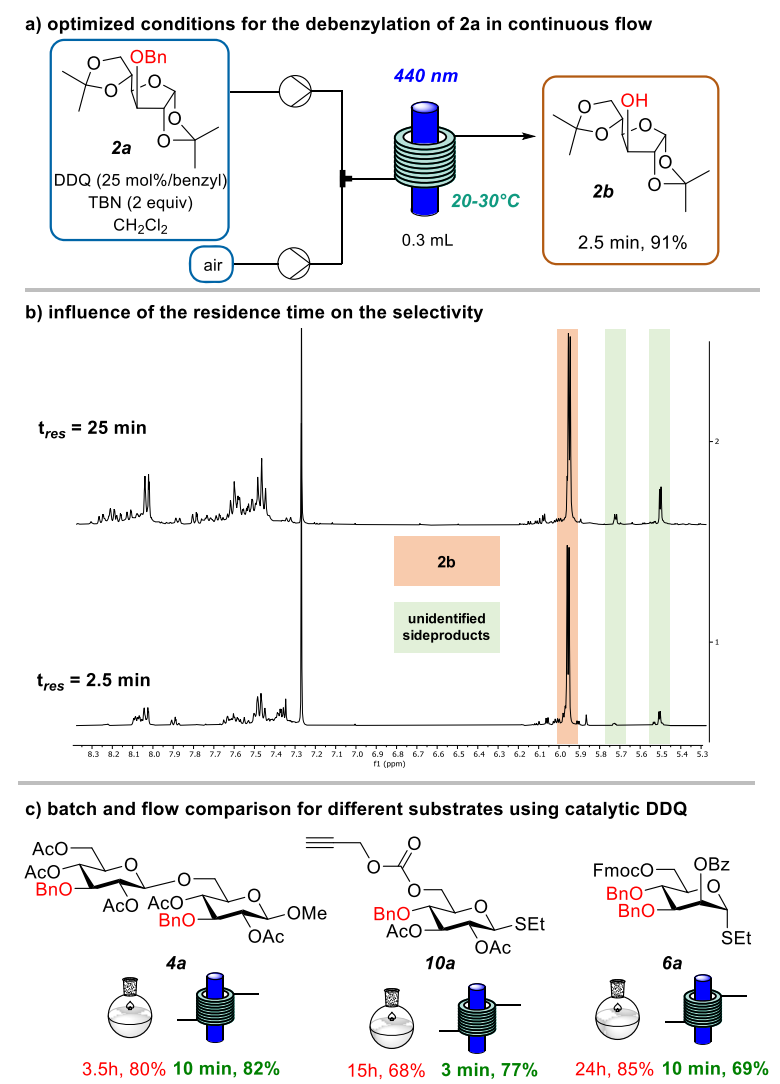

Figure 2. Visible-light-mediated oxidative cleavage of benzyl ethers using a continuous-flow system.

continuous-flow system helped to reduce the reaction times. Precise control of the reaction time and efficient irradiation in 
flow enabled the use of $440 \mathrm{~nm}$ to significantly reduce reaction times while maintaining high selectivities. The photooxidative debenzylation overcomes the current limitations of benzyl ethers as protecting groups that arise from the harsh conditions necessary for their cleavage. The methodology enables the use of benzyl ethers as a temporary protective group and is attractive for the development of new synthetic routes in glycan synthesis.

\section{ASSOCIATED CONTENT}

\section{Supporting Information}

The Supporting Information is available free of charge at https://pubs.acs.org/doi/10.1021/acs.orglett.0c04026.

Detailed experimental procedures and characterization data for all compounds (PDF)

\section{AUTHOR INFORMATION}

\section{Corresponding Authors}

Bartholomäus Pieber - Department of Biomolecular Systems, Max Planck Institute of Colloids and Interfaces, 14476 Potsdam, Germany; 이이.org/0000-0001-8689-388X; Email: bartholomaeus.pieber@mpikg.mpg.de

Peter H. Seeberger - Department of Biomolecular Systems, Max Planck Institute of Colloids and Interfaces, 14476 Potsdam, Germany; Department of Chemistry and Biochemistry, Freie Universität Berlin, 14195 Berlin, Germany; Email: peter.seeberger@mpikg.mpg.de

\section{Authors}

Cristian Cavedon - Department of Biomolecular Systems, Max Planck Institute of Colloids and Interfaces, 14476 Potsdam, Germany; Department of Chemistry and Biochemistry, Freie Universität Berlin, 14195 Berlin, Germany

Eric T. Sletten - Department of Biomolecular Systems, Max Planck Institute of Colloids and Interfaces, 14476 Potsdam, Germany

Amiera Madani - Department of Biomolecular Systems, Max Planck Institute of Colloids and Interfaces, 14476 Potsdam, Germany; Department of Chemistry and Biochemistry, Freie Universität Berlin, 14195 Berlin, Germany

Olaf Niemeyer - Department of Biomolecular Systems, Max Planck Institute of Colloids and Interfaces, 14476 Potsdam, Germany

Complete contact information is available at:

https://pubs.acs.org/10.1021/acs.orglett.0c04026

\section{Author Contributions}

The manuscript was written through contributions of all authors. All authors have given approval to the final version of the manuscript.

Notes

A preprint of this research was previously posted to ChemRxiv on October 27, 2020: Cavedon, C.; Sletten, E. T.; Madani, A.; Niemeyer, O.; Seeberger, P. H.; Pieber, B. Visible LightMediated Oxidative Debenzylation. ChemRxiv 2020, DOI: 10. 26434/chemrxiv.13135814.

The authors declare no competing financial interest.

\section{ACKNOWLEDGMENTS}

We gratefully acknowledge the Max-Planck Society for generous financial support. A.M. and B.P. acknowledge the Deutsche Forschungsgemeinschaft (DFG, German Research Foundation) under Germany's Excellence Strategy-EXC 2008/1 (UniSysCat)-390540038 for financial support. B.P. acknowledges financial support from a Liebig Fellowship of the German Chemical Industry Fund (Fonds der Chemischen Industrie, FCI). E.T.S. acknowledges financial support from the Alexander von Humboldt Foundation.

\section{REFERENCES}

(1) Greene, T. W.; Wuts, P. G. M. Protective Groups in Organic Synthesis, 3rd ed.; Wiley: New York, 1999.

(2) Wang, T.; Demchenko, A. V. Synthesis of carbohydrate building blocks via regioselective uniform protection/deprotection strategies. Org. Biomol. Chem. 2019, 17, 4934-4950.

(3) Krasnova, L.; Wong, C.-H. Oligosaccharide Synthesis and Translational Innovation. J. Am. Chem. Soc. 2019, 141, 3735-3754.

(4) Guberman, M.; Seeberger, P. H. Automated Glycan Assembly: A Perspective. J. Am. Chem. Soc. 2019, 141, 5581-5592.

(5) Crawford, C.; Oscarson, S. Optimized Conditions for the Palladium-Catalyzed Hydrogenolysis of Benzyl and Naphthylmethyl Ethers: Preventing Saturation of Aromatic Protecting Groups. Eur. J. Org. Chem. 2020, 2020, 3332-3337.

(6) Van Ornum, S. G.; Champeau, R. M.; Pariza, R. Ozonolysis Applications in Drug Synthesis. Chem. Rev. 2006, 106, 2990-3001.

(7) Joshi, D. K.; Sutton, J. W.; Carver, S.; Blanchard, J. P. Experiences with Commercial Production Scale Operation of Dissolving Metal Reduction Using Lithium Metal and Liquid Ammonia. Org. Process Res. Dev. 2005, 9, 997-1002.

(8) Horita, K.; Yoshioka, T.; Tanaka, T.; Oikawa, Y.; Yonemitsu, O. On the selectivity of deprotection of benzyl, mpm (4-methoxybenzyl) and dmpm (3,4-dimethoxybenzyl) protecting groups for hydroxy functions. Tetrahedron 1986, 42, 3021-3028.

(9) Classon, B.; Garegg, P. J.; Samuelsson, B.; Lawesson, S.-O.; Norin, T. The p-Methoxybenzyl Group as Protective Group of the Anomeric Centre. Selective Conversions of Hydroxy Groups into Bromo Groups in p-Methoxybenzyl 2-Deoxy-2-phthalimido-beta-Dglucopyranoside. Acta Chem. Scand. 1984, 38b, 419-422.

(10) Vaino, A. R.; Szarek, W. A. Iodine in Methanol: a Simple Selective Method for the Cleavage of p-Methoxybenzyl Ethers. Synlett 1995, 1995, 1157-1158.

(11) Tucker, J. W.; Narayanam, J. M. R.; Shah, P. S.; Stephenson, C. R. J. Oxidative photoredox catalysis: mild and selective deprotection of PMB ethers mediated by visible light. Chem. Commun. 2011, 47, $5040-5042$.

(12) Liu, Z.; Zhang, Y.; Cai, Z.; Sun, H.; Cheng, X. Photoredox Removal of p-Methoxybenzyl Ether Protecting Group with Hydrogen Peroxide as Terminal Oxidant. Adv. Synth. Catal. 2015, 357, 589593.

(13) Ahn, D. K.; Kang, Y. W.; Woo, S. K. Oxidative Deprotection of p-Methoxybenzyl Ethers via Metal-Free Photoredox Catalysis. J. Org. Chem. 2019, 84, 3612-3623.

(14) Mayeda, E. A.; Miller, L. L.; Wolf, J. F. Electrooxidation of benzylic ethers, esters, alcohols, and phenyl epoxides. J. Am. Chem. Soc. 1972, 94, 6812-6816.

(15) Ohkubo, K.; Fujimoto, A.; Fukuzumi, S. Visible-Light-Induced Oxygenation of Benzene by the Triplet Excited State of 2,3-Dichloro5,6-dicyano-p-benzoquinone. J. Am. Chem. Soc. 2013, 135, 53685371.

(16) For a discussion of possible mechanisms, see the Supporting Information.

(17) Rahim, M. A.; Matsumura, S.; Toshima, K. Deprotection of benzyl ethers using 2,3-dichloro-5,6-dicyano-p-benzoquinone (DDQ) under photoirradiation. Tetrahedron Lett. 2005, 46, 7307-7309. 
(18) Song, C.; Yi, H.; Dou, B.; Li, Y.; Singh, A. K.; Lei, A. Visiblelight-mediated $\mathrm{C} 2$-amination of thiophenes by using DDQ as an organophotocatalyst. Chem. Commun. 2017, 53, 3689-3692.

(19) Rusch, F.; Schober, J.-C.; Brasholz, M. Visible-Light Photocatalytic Aerobic Benzylic $\mathrm{C}(\mathrm{sp} 3)-\mathrm{H}$ Oxygenations with the $3 \mathrm{DDQ}^{*} /$ tert-Butyl Nitrite Co-catalytic System. ChemCatChem 2016, 8, 2881-2884.

(20) Wang, Y.; Wang, S.; Chen, B.; Li, M.; Hu, X.; Hu, B.; Jin, L.; Sun, N.; Shen, Z. Visible-Light-Induced Arene C(sp2)-H Lactonization Promoted by DDQ and tert-Butyl Nitrite. Synlett 2020, 31, 261266.

(21) Das, S.; Natarajan, P.; König, B. Teaching Old Compounds New Tricks: DDQ-Photocatalyzed $\mathrm{C}-\mathrm{H}$ Amination of Arenes with Carbamates, Urea, and N-Heterocycles. Chem. - Eur. J. 2017, 23, 18161-18165.

(22) Pan, D.; Pan, Z.; Hu, Z.; Li, M.; Hu, X.; Jin, L.; Sun, N.; Hu, B.; Shen, Z. Metal-Free Aerobic Oxidative C-O Coupling of C(sp3)-H with Carboxylic Acids Catalyzed by DDQ and tert-Butyl Nitrite. Eur. J. Org. Chem. 2019, 2019, 5650-5655.

(23) Song, C.; Dong, X.; Yi, H.; Chiang, C.-W.; Lei, A. DDQCatalyzed Direct C(sp3)-H Amination of Alkylheteroarenes: Synthesis of Biheteroarenes under Aerobic and Metal-Free Conditions. ACS Catal. 2018, 8, 2195-2199.

(24) Shen, Z.; Dai, J.; Xiong, J.; He, X.; Mo, W.; Hu, B.; Sun, N.; Hu, X. 2,3-Dichloro-5,6-dicyano-1,4-benzoquinone (DDQ)/tert-Butyl Nitrite/Oxygen: A Versatile Catalytic Oxidation System. Adv. Synth. Catal. 2011, 353, 3031-3038.

(25) Shen, Z.; Sheng, L.; Zhang, X.; Mo, W.; Hu, B.; Sun, N.; Hu, X. Aerobic oxidative deprotection of benzyl-type ethers under atmospheric pressure catalyzed by 2,3-dichloro-5,6-dicyano-1,4-benzoquinone (DDQ)/tert-butyl nitrite. Tetrahedron Lett. 2013, 54, 15791583.

(26) Feldmeier, C.; Bartling, H.; Riedle, E.; Gschwind, R. M. LED based NMR illumination device for mechanistic studies on photochemical reactions - Versatile and simple, yet surprisingly powerful. J. Magn. Reson. 2013, 232, 39-44.

(27) Tanemura, K.; Suzuki, T.; Horaguchi, T. Deprotection of silyl ethers using 2,3-dichloro-5,6-dicyano-p-benzoquinone. J. Chem. Soc., Perkin Trans. 1 1992, 22, 2997-2998.

(28) Plutschack, M. B.; Pieber, B.; Gilmore, K.; Seeberger, P. H. The Hitchhiker's Guide to Flow Chemistry. Chem. Rev. 2017, 117, 11796-11893.

(29) Cambié, D.; Bottecchia, C.; Straathof, N. J. W.; Hessel, V.; Noël, T. Applications of Continuous-Flow Photochemistry in Organic Synthesis, Material Science, and Water Treatment. Chem. Rev. 2016, 116, 10276-10341. 